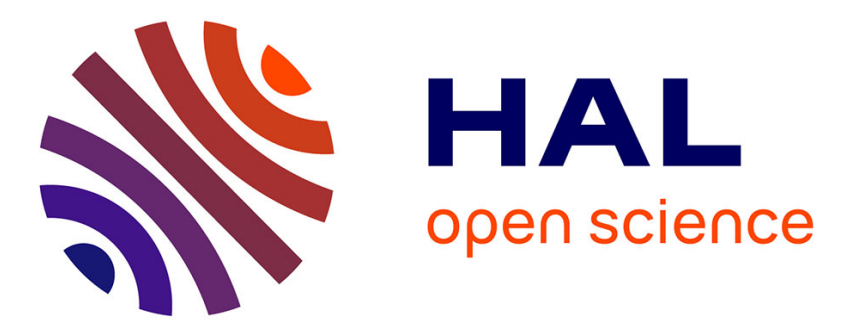

\title{
A methodology for an accurate evaluation of the linearization procedures in nonlinear mean field homogenization
}

Amna Rekik, Michel Bornert, François Auslender, André Zaoui

\section{To cite this version:}

Amna Rekik, Michel Bornert, François Auslender, André Zaoui. A methodology for an accurate evaluation of the linearization procedures in nonlinear mean field homogenization. Comptes Rendus Mécanique, 2005, 333, pp.789-795. 10.1016/j.crme.2005.10.002 . hal-00156073

\section{HAL Id: hal-00156073 https://hal.science/hal-00156073}

Submitted on 16 Sep 2021

HAL is a multi-disciplinary open access archive for the deposit and dissemination of scientific research documents, whether they are published or not. The documents may come from teaching and research institutions in France or abroad, or from public or private research centers.
L'archive ouverte pluridisciplinaire HAL, est destinée au dépôt et à la diffusion de documents scientifiques de niveau recherche, publiés ou non, émanant des établissements d'enseignement et de recherche français ou étrangers, des laboratoires publics ou privés.

\section{(c)(1)}

Distributed under a Creative Commons Attribution| 4.0 International License 


\title{
A methodology for an accurate evaluation of the linearization procedures in nonlinear mean field homogenization
}

\author{
Amna Rekik ${ }^{a}$, Michel Bornert ${ }^{\mathrm{a}}$, François Auslender ${ }^{\mathrm{a}, \mathrm{b}}$, André Zaoui ${ }^{\mathrm{a}, *}$ \\ a Laboratoire de mécanique des solides, École polytechnique, CNRS UMR 7649, 91128 Palaiseau cedex, France \\ ${ }^{\mathrm{b}}$ Laboratoire de mécanique et ingénieries, université Blaise Pascal/IFMA, 24, avenue des landais, 63170 Aubière, France
}

\begin{abstract}
A systematic methodology for the evaluation of the linearization procedures sustaining mean field homogenization theories for nonlinear composite materials is proposed and applied as an illustration to various recently proposed 'affine' and 'second-order' formulations for nonlinear elasticity. It relies on the analysis of composites for which both the exact nonlinear homogenization problem and the homogenization problem associated with the 'linear comparison material' defined by the linearization procedure can be solved numerically with the same accuracy and for the same microstructure. The comparison of the results then provides a rigorous evaluation of the effects of the sole linearization method.
\end{abstract}

Keywords: Continuum mechanics; Homogenization; Nonlinear behavior; Linearization; Affine; Second order

\section{Résumé}

Une méthodologie pour l'évaluation précise des procédures de linéarisation en homogénéisation non linéaire en champs moyens. Une méthodologie systématique pour l'évaluation des procédures de linéarisation sur lesquelles s'appuient les théories d'homogénéisation en champs moyens pour les composites non linéaires est présentée et appliquée à titre d'illustration pour l'élasticité non linéaire aux différentes variantes des formulations « affine » et « au second ordre » récemment proposées. Elle repose sur l'analyse de composites pour lesquels on résout numériquement avec la même précision et pour la même microstructure aussi bien le problème d'homogénéisation non linéaire complet que le problème d'homogénéisation linéaire relatif au «milieu linéaire de comparaison » défini par la procédure de linéarisation. La comparaison des résultats permet ainsi d'évaluer rigoureusement les seuls effets de la méthode de linéarisation.

Mots-clés : Milieux continus ; Homogénéisation ; Comportement non linéaire ; Linéarisation ; Affine ; Second ordre

\footnotetext{
* Corresponding author.

E-mail addresses: rekik@1ms.polytechnique.fr (A. Rekik), bornert@1ms.polytechnique.fr (M. Bornert), auslende@1ms.polytechnique.fr (F. Auslender), zaoui@1ms.polytechnique.fr (A. Zaoui).
} 


\section{Introduction}

Most homogenization theories for nonlinear heterogeneous random materials (for a review, refer to [1]) explicitly or implicitly rely on a two-stage formulation. The first stage amounts to replacing the nonlinear equations with some linear or affine relations through a so-called linearization procedure. The nonlinearities generally stem from the material behavior and sometimes from the kinematics when a large strain framework is used. The linear or affine relations depend on parameters which are constant per phase and evolve with the overall load. The system of linear or affine equations derived from the linearization procedure corresponds to a homogenization problem associated with a fictitious elastic or thermoelastic composite, usually referred to as the linear comparison composite (LCC). The second stage consists in solving these linear equations by means of an upscaling model, appropriate for the random microstructure of the LCC. If, at each stage, the derivation ensures that the result is a bound for the effective properties [2], the derived global estimate is also a bound. Unfortunately, such a bound often does not provide a sufficiently accurate approximation of the real effective behavior. Some recent linearization procedures, like the affine approach [3], the second-order procedure [4,5], and some of their variants [6-8] seem to be more efficient. Nevertheless, their relevance and their relative performance need to be more precisely assessed.

To this end, the prediction of these formulations may be compared, either with the few efficient and stringent bounds available for some particular cases [9], or with the expansions exact to second order in the contrast available for weakly inhomogeneous nonlinear composites [10]. For more general morphologies or in situations where the contrast between the phases is not small, the comparison with full field numerical solutions of the initial nonlinear homogenization problem is the only possible way to evaluate the accuracy of the predictions of mean fields theories. To this end, two different approaches may be considered. The first consists in carrying out numerical simulations on large windows of simulated microstructures [11] supposed to mimic the random microstructures addressed by the mean field theories used for the homogenization of the LCC. With such an approach, one has to face computational difficulties due to the large size of the numerical system to work out, as well as questions relative to the statistical representativeness of the generated microstructures, the appropriate averaging of the results and the choice of particular boundary conditions [12]. Due to current computer power limitations, this first approach is often restricted either to two-dimensional problems or, when three-dimensional simulations are required, to a restricted number of comparisons which does not allow us to exhaustively explore all combinations of the parameters of the particular problem under consideration. In the second approach, predictions of nonlinear mean field theories are compared to numerical simulations on simple periodic microstructures, for which the computational expense or the control of the numerical accuracy of the results are no longer an issue $[13,7]$. However, in such a case, the comparison between the numerical solution and a nonlinear mean field theory based on a linear model for a random LCC is strongly altered by the fact that both approaches do not describe the same microstructure.

In both situations, the comparisons are also distorted by the fact that the mean field models often rely on the use of linear closed form estimates to evaluate the behavior of the LCC, which are, in general, not exact results. Such linear estimates might not be sufficiently accurate, especially in the case of complex microstructures and high contrast. Accordingly, the conclusions may be potentially ambiguous because of the addition of both approximations, induced first by the linearization procedure itself and second by the linear homogenization scheme, which may be cumulative or compensating.

\section{Proposed methodology}

The methodology and the numerical tool which are proposed in this paper enable a systematic and accurate evaluation of the linearization procedures which is not restricted by the aforementioned limitations. To this end, we address a problem where the nonlinear exact solution, regarded as the reference solution, may be computed numerically with high accuracy at a low computational expense. Moreover, the homogenization of the LCC, which retains the microstructure of the nonlinear composite, is carried out by the same numerical tool as the one handling the nonlinear composite. Thereby, the difficulties related to the numerical approximations, to the change of microstructure as well as to the approximations induced by the linear estimates of the LCC are avoided. The effect of the sole linearization procedure may be assessed without any ambiguity. In concrete terms, the chosen microstructure is periodic and classical finite element techniques associated with periodic homogenization are used to solve both the initial nonlinear problem and the linear one associated with the LCC. Although the proposed methodology is not completely exhaustive, it 
allows us to handle large classes of problems, whatever the choices of the local constitutive laws, the morphology of the periodic microstructure and the type of solicitation.

To illustrate the proposed methodology, we consider the particular case of composites for which the constitutive behavior of the individual constituents is elastic and governed by a single potential or strain energy function and the classical small strain framework is used. This situation is formally similar to the rate problem for a nonlinear viscoplastic composite. For the sake of simplicity, the studied material reduces to a two-phase composite, composed of identical isotropic linear elastic spherical inclusions embedded in a nonlinear isotropic matrix, the constitutive relation of which relates the stress $\sigma$ to the strain $\varepsilon$ according to $\sigma=\frac{\partial \omega^{m}}{\partial \varepsilon}(\varepsilon)=f^{m}(\varepsilon)$, where $\omega^{m}$ is the strain potential of the matrix. In addition, the matrix is classically assumed to behave nonlinearly only in its deviatoric part:

$$
\sigma_{m}=3 k^{m} \varepsilon_{m} \quad \text { and } \quad \sigma_{\mathrm{eq}}=\frac{\partial \omega_{\mathrm{eq}}^{m}}{\partial \varepsilon}=f_{\mathrm{eq}}^{m}\left(\varepsilon_{\mathrm{eq}}\right)
$$

where $\sigma_{\mathrm{eq}}=\sqrt{\frac{3}{2} \sigma \mathbf{K} \sigma}$ and $\varepsilon_{\mathrm{eq}}=\sqrt{\frac{2}{3} \varepsilon \mathbf{K} \varepsilon}$ are the von Mises equivalents of the stress and strain tensors, $\sigma_{m}=\frac{1}{3} i: \sigma$ and $\varepsilon_{m}=\frac{1}{3} i: \varepsilon$ are their spherical parts. In these relations, $i$ is the second-order identity tensor and $\mathbf{K}=\mathbf{I}-\mathbf{J}$ with $\mathbf{I}$ the fourth-order identity tensor and $\mathbf{J}=\frac{1}{3} i \otimes i$. Note that, unlike many previous studies, the behavior is compressible (the bulk modulus $k^{m}$ is finite).

The spherical inclusions are distributed according to a hexagonal network in the transverse plane and are aligned along the third direction, such that a cylinder with a hexagonal basis with a single spherical inclusion can be used as unit cell. The loading conditions that will be considered are axial symmetric along the third direction. In a first and classical approximation [14], this hexagonal cell can be replaced by a cylinder with a circular basis, making the whole unit cell problem fully invariant with respect to any rotation along this direction. This 3D homogenization problem then reduces to a 2D axial symmetrical problem which can be solved at a low numerical cost. The unit cell being in addition symmetric with respect to the transverse plane, only one fourth of the cross section of the cylinder needs to be meshed. Symmetry conditions are prescribed on the transverse plane and along the symmetry axis and homogeneous longitudinal or radial displacements are imposed on the upper or lateral face, respectively.

In the ensuing calculations, the unit cell is submitted to a monotonic uniaxial purely deviatoric extension along the symmetry axis, such that the overall deformation is $\bar{\varepsilon}=\bar{\varepsilon}_{\mathrm{eq}} \hat{e}$ with $\hat{e}=e_{3} \otimes e_{3}-\frac{1}{2}\left(e_{2} \otimes e_{2}+e_{1} \otimes e_{1}\right)$. For symmetry reasons, the overall stress is then $\bar{\sigma}=\frac{2}{3} \bar{\sigma}_{\mathrm{eq}} \hat{e}+\bar{\sigma}_{m} i$. We focus our attention on the effective deviatoric response $\bar{\sigma}_{\text {eq }}=\tilde{f}\left(\bar{\varepsilon}_{\text {eq }}\right)$. Note that, as a consequence of rotational invariance of the problem, the only non zero components of the overall stress and strain tensors are the diagonal ones, with component 11 equal to component 22 , so that $\bar{\sigma}_{\text {eq }}=\left|\bar{\sigma}_{33}-\bar{\sigma}_{11}\right|$ and $\bar{\varepsilon}_{\text {eq }}=\frac{2}{3}\left|\bar{\varepsilon}_{33}-\bar{\varepsilon}_{11}\right|$.

The nonlinear solution referred to as NL is obtained through finite element calculations carried out with the finite element code Cast3M. The linearization procedures which are described in Section 3 are implemented in C++ routines. The effective properties and the local responses of the LCC are derived by means of additional finite element calculations based on the same mesh and the same symmetry and periodicity conditions. They are obtained with Cast3M as well, which has been appropriately interfaced with the $\mathrm{C}++$ programs.

\section{Nonlinear formulations}

For all formulations, the inclusions of the LCC behave like the linear elastic inclusions of the nonlinear composite. Let $L^{p}$ be their tensor of elastic moduli and $f^{p}$ their volume fraction. The matrix of the LCC follows a thermoelastic constitutive law $\sigma=L^{m} \varepsilon+\tau^{m}$, with a uniform tensor of moduli $L^{m}$ and a polarization stress $\tau^{m}$. These quantities are determined by equations which are invariant with respect to any rotation along the third axis. Accordingly, $L^{m}$ is at least transversely isotropic with respect to this axis; it is even isotropic for specific formulations. As a consequence, the tensor $\tilde{L}$ of effective moduli of the LCC is transversely isotropic and can be cast in the following form:

$$
\tilde{L}=\tilde{\alpha} \mathbf{E}^{L}+\tilde{\beta} \mathbf{J}^{T}+\tilde{\gamma} \mathbf{F}+\tilde{\gamma}^{\prime}{ }^{t} \mathbf{F}+\tilde{\delta} \mathbf{K}^{T}+\tilde{\delta}^{\prime} \mathbf{K}^{L}
$$

where the fourth-order tensors $\mathbf{F}, \mathbf{E}^{L}, \mathbf{J}^{T}, \mathbf{K}^{T}$ and $\mathbf{K}^{L}$, introduced by Walpole [15], are defined in [16]. Finite elements calculations performed for two independent axial symmetric loadings (uniaxial tension and transverse compression for instance) provide $\tilde{\alpha}, \tilde{\beta}$ and $\tilde{\gamma}=\tilde{\gamma}^{\prime}$. The moduli $\tilde{\delta}$ and $\tilde{\delta}^{\prime}$ can be derived from a Fourier analysis in mode 1 (longitudinal shear) and 2 (transverse shear) but are not necessary in the present study. The spherical part of $\tau^{m}$ being always zero, 
this tensor is proportional to $\hat{e}$. The classical Levin relations for two-phase thermoelastic composites provide the effective polarization $\tilde{\tau}$.

The constitutive law of the LCC is derived from the nonlinear constitutive law of the matrix (1), from the strain average $\bar{\varepsilon}^{m}=\langle\varepsilon\rangle_{m}$ inside the matrix of the LCC and, for some formulations, from the covariance tensor $C_{\varepsilon}^{m}=\langle(\varepsilon-$ $\left.\left.\bar{\varepsilon}^{m}\right) \otimes\left(\varepsilon-\bar{\varepsilon}^{m}\right)\right\rangle_{m}=\langle\varepsilon \otimes \varepsilon\rangle_{m}-\bar{\varepsilon}^{m} \otimes \bar{\varepsilon}^{m}$ which characterizes the strain field fluctuations inside the matrix. When the LCC is linear elastic ( $\tau^{m}=0$ ), the localization relation $\varepsilon(x)=A(x) \bar{\varepsilon}$ yields the matrix average strain $\langle\varepsilon\rangle_{m}=\langle A\rangle_{m} \bar{\varepsilon}=$ $A^{m} \bar{\varepsilon}$. The localization tensor $A^{m}$ is transversely isotropic but not symmetric and admits a decomposition similar to (2). It can be directly derived from $\tilde{L}, L^{p}, L^{m}$ and $f^{p}$. Alternatively, it can also be identified by direct integrations over the matrix of the computed local solutions used to determine $\tilde{L}$. The second-order moment of the strain in the matrix can be computed [13] by derivating the effective energy with respect to the local moduli $\langle\varepsilon \otimes \varepsilon\rangle_{m}=\frac{1}{1-f^{p}} \bar{\varepsilon} \frac{\partial \tilde{L}}{\partial L^{m}} \bar{\varepsilon}$. However, the computational expense of such an operation is high; in the present case a direct numerical integration of the local strain fields provided by the finite element calculations is a more efficient procedure. Note that $\langle\varepsilon \otimes \varepsilon\rangle_{m}$ is a fourth-order transversely isotropic symmetric tensor; three of its five constants defined by (2) are required for the implementation of the considered nonlinear extensions. For a thermoelastic LCC, the local strain field is given by Levin's relation:

$$
\varepsilon(x)=A(x) \bar{\varepsilon}+(A(x)-I)\left(L^{m}-L^{p}\right)^{-1} \tau^{m}
$$

A simple averaging of this equation provides the required average strain in the matrix, as well as its second-order moment. The obtained expressions, which are not detailed here, give these quantities as functions of their counterparts in the purely elastic case.

The full effective stress-strain curve is constructed pointwise for several prescribed overall deformations. At each step, a classical fixed point iterative procedure is used to solve the nonlinear equations which describe the LCC. An initial approximation of the matrix behavior is set, such as for instance the initial linear elastic behavior or the solution at previous step. Then, the first and second moments of the matrix local fields are computed and provide a new approximation of the matrix thermoelastic behavior according to the linearization procedures described hereafter. The procedure is repeated until convergence.

\subsection{Variational formulation (VAR)}

For this approach also referred to as the modified secant extension [1], the LCC is elastic $\left(\tau^{m}=0\right)$. The modulus $L^{m}$ is defined as the isotropic secant modulus evaluated at the second order moment of the matrix strain field:

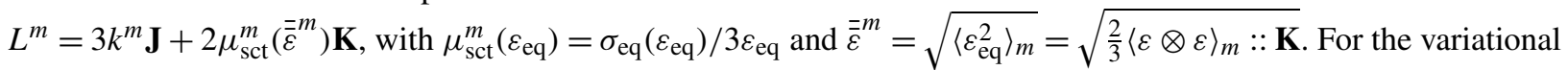
formulation, the local solution of the nonlinear composite is approximated by the solution of the LCC. Therefore, the macroscopic stress can be determined by $\bar{\sigma}=\tilde{L}: \bar{\varepsilon}$. It is noteworthy that this formulation provides an upper bound for the effective energy.

\subsection{Affine formulations}

According to the original affine approach AFF-ANI, the linear thermoelastic behavior of the matrix in the LCC is given by the tangent to its nonlinear matrix at the matrix average strain [3]: $L^{m}=L_{\text {tgt }}^{m}\left(\bar{\varepsilon}^{m}\right)=\frac{\partial^{2} w^{m}}{\partial \varepsilon^{2}}\left(\bar{\varepsilon}^{m}\right)$ and $\tau^{m}=$ $\frac{\partial w^{m}}{\partial \varepsilon}\left(\bar{\varepsilon}^{m}\right)-L^{m}: \bar{\varepsilon}^{m}$. Unlike the variational approach, the tensor $L^{m}$ is transversely isotropic and can be written as $3 k^{m} \mathbf{J}+2 \mu_{\mathrm{sct}}^{m}\left(\bar{\varepsilon}_{\mathrm{eq}}^{m}\right) \mathbf{F}+2 \mu_{\mathrm{tgt}}^{m}\left(\bar{\varepsilon}_{\mathrm{eq}}^{m}\right) \mathbf{E}$, where $\mu_{\mathrm{tgt}}^{m}\left(\varepsilon_{\mathrm{eq}}\right)=\frac{d \sigma_{\mathrm{eq}}}{3 d \varepsilon_{\mathrm{eq}}}\left(\varepsilon_{\mathrm{eq}}\right)$ is the tangent shear modulus, $\mathbf{E}=\frac{2}{3} \hat{e} \otimes \hat{e}$ and $\mathbf{F}=\mathbf{K}-\mathbf{E}$ are the classical fourth-order projectors [1] relative to the directions parallel and perpendicular to the overall load. Like the variational approach, the local solution of the nonlinear composite is approximated by the solution of the LCC and the macroscopic stress is similarly derived by $\bar{\sigma}=\tilde{L}: \bar{\varepsilon}+\tilde{\tau}$. Due to the anisotropy of $L^{m}$, the affine formulation is more difficult to apply to practical situations than the variational formulation. To get round this drawback, two simplified isotropic versions are proposed. In the first variant referred to as AFF-ISOT, the $L^{m}$ modulus tensor is defined as $L^{m}=3 k^{m} \mathbf{J}+2 \mu_{\mathrm{tgt}}^{m}\left(\bar{\varepsilon}_{\mathrm{eq}}^{m}\right) \mathbf{K}$ as proposed in [7]. In the second variant AFF-ISOI, the tensor of moduli is defined as the projection of the actual transversely isotropic tangent tensor on the subspace of isotropic tensors: $L^{m}=3 k^{m} \mathbf{J}+2 \mu_{\mathrm{inv}}^{m}\left(\bar{\varepsilon}_{\mathrm{eq}}^{m}\right) \mathbf{K}$ with $\mu_{\mathrm{inv}}^{m}=\frac{4 \mu_{\mathrm{sct}}^{m}+\mu_{\mathrm{tgt}}^{m}}{5}$. 


\subsection{Second-order formulations}

The initial second-order estimate, referred to as SOE-1, is based on a second-order Taylor expansion [4] of the strain potential $w^{r}$ in each phase $r(r=1,2)$ around a reference strain $\bar{\varepsilon}^{r}$ equal to the strain average over the considered phase. While the derived LCC is exactly the same as the one of the affine formulation, the method to evaluate the effective response is different and relies on the construction of an effective potential estimated by $\widetilde{w}(\bar{\varepsilon}) \approx \sum_{r} f_{r}\left(w^{r}\left(\bar{\varepsilon}^{r}\right)+\frac{1}{2} \frac{\partial w}{\partial \varepsilon}\left(\bar{\varepsilon}^{r}\right):\left(\bar{\varepsilon}-\bar{\varepsilon}^{r}\right)\right)$, from which the effective stress derives according to $\bar{\sigma}=\frac{\partial \widetilde{w}}{\partial \bar{\varepsilon}}(\bar{\varepsilon})$. As pointed out in Section 2, we only need to calculate $\bar{\sigma}_{\text {eq }}=\frac{\partial \widetilde{w}}{\partial \bar{\varepsilon}_{\mathrm{eq}}}\left(\bar{\varepsilon}_{\mathrm{eq}}\right)$. This derivation is performed numerically. The discrepancies between the affine and initial second-order formulations are associated with the intra-phase fluctuations of the local fields [5]. The estimated local strain fields are the same in both formulations, but the definition of the local stress field in the SOE-1 formulation remains ambiguous. However, an estimate of the local stress field can be rigorously defined from the dual version of the SOE-1 formulation which relies on a stress (instead of strain) energy function [4].

In the new improved second-order formulation SOE-2, based on stationarity conditions related to the evaluated effective energy [6], $L^{m}$ is defined by means of a generalized secant relation $\frac{\partial w^{m}}{\partial \varepsilon}\left(\hat{\varepsilon}^{m}\right)-\frac{\partial w^{m}}{\partial \varepsilon}\left(\bar{\varepsilon}^{m}\right)=L^{m}:\left(\hat{\varepsilon}^{m}-\right.$ $\left.\bar{\varepsilon}^{m}\right)$. The tensor $L^{m}$ is still expressed as a combination of tensors $\mathbf{J}, \mathbf{E}$ and $\mathbf{F}$. The additional reference strain $\hat{\varepsilon}^{m}$ used to characterize intraphase strain fluctuations can be split into two components according to the relation $\hat{\varepsilon}^{m}=$ $\hat{\varepsilon}_{\|}^{m} \hat{e}+\hat{\varepsilon}_{\perp}^{m}$. Each component can be assessed from the covariance tensor $C_{\varepsilon}^{m}$ by means of the following relations $\hat{\varepsilon}_{\|}^{m}=\bar{\varepsilon}_{\text {eq }}^{m}+\sqrt{\frac{2}{3} \mathbf{E}:: C_{\varepsilon}^{m}}$ and $\hat{\varepsilon}_{\perp \text { eq }}^{m}=\sqrt{\frac{2}{3} \mathbf{F}:: C_{\varepsilon}^{m}}$. Unlike the aforementioned formulations AFF-ANI and SOE-1, the SOE-2 procedure explicitly accounts for the intra-phase strain field fluctuations in the definition of the LCC. At last, it is worth-noting that a dual version of the second-order procedures based on the stress potential $u(\sigma)-$ i.e., the Legendre transform of the strain potential $w(\varepsilon)$ - is available in $[4,6]$. In the sequel, for the sake of simplicity, we will only consider the second-order procedures based on the strain potential $w(\varepsilon)$.

\subsection{Lahellec-Suquet formulation ( $\mathbf{S}$ )}

This new formulation [8] retains the energetic framework of the initial second order estimate and modifies it in such a way that the field formulation $(\bar{\sigma}=\langle\sigma\rangle)$ is in precise agreement with the energetic formulation $\left(\bar{\sigma}=\frac{\partial \widetilde{w}}{\partial \bar{\varepsilon}}\right)$. To this end, the matrix strain potential $w^{m}$ is approximated by a third order Taylor expansion estimated at $\bar{\varepsilon}^{m}=\langle\varepsilon\rangle_{m}$. Then, the cubic term is itself approximated by a linear term which allows for the local strain field fluctuations. The LCC moduli can then be expressed:

$$
L^{m}=L_{\text {tgt }}^{m}\left(\bar{\varepsilon}^{m}\right) \quad \text { and } \quad \tau^{m}=\frac{\partial w^{m}}{\partial \varepsilon}\left(\bar{\varepsilon}^{m}\right)-L^{m}: \bar{\varepsilon}^{m}+\frac{1}{2} N^{m}\left(\bar{\varepsilon}^{m}\right):: C_{\varepsilon}^{m} \quad \text { where } N^{m}(\varepsilon)=\frac{\partial^{3} w^{m}}{\partial \varepsilon^{3}}(\varepsilon)
$$

The main difference between this new approach and the AFF-ANI and SOE-1 approaches lies in the polarization $\tau^{m}$ which generates a 'softer' LCC in the LS formulation. As in the affine approach, the local fields of the nonlinear composite are approximated by the local fields of the LCC.

\section{Results, comments and perspectives}

The present methodology is carried out on a two-phase reinforced composite the material parameters of which are identical to those defined in [7]. The matrix follows a Ramberg-Osgood type constitutive law, with a threshold and an initial isotropic elastic behavior:

$$
\varepsilon_{\mathrm{eq}}=\frac{\sigma_{\mathrm{eq}}}{3 \mu^{m}}+\varepsilon_{0}\left(\frac{P\left(\sigma_{\mathrm{eq}}-\sigma_{y}\right)}{\sigma_{0}}\right)^{n}
$$

where $m=1 / n$ is the strain-hardening parameter ( $n$ is the nonlinearity exponent) such that $0 \leqslant m \leqslant 1, \sigma_{0}$ is the flow stress, $\sigma_{y}$ is the threshold stress, $\varepsilon_{0}$ is an auxiliary strain and $P(a)$ is the positive part of $a$. The nonlinear part of the matrix constitutive law is taken into account as soon as $\sigma_{y}=3 \mu^{m} \varepsilon_{\text {ref }}$ where $\varepsilon_{\text {ref }}$ denotes one of the considered reference strains. The macroscopic responses $\bar{\sigma}_{\text {eq }}=\tilde{f}\left(\bar{\varepsilon}_{\mathrm{eq}}\right)$ obtained with the formulations depicted above are reported in Fig. 1. The AFF-ANI approach associated with the matrix strain average is too stiff as already noticed in [3,9]. It 

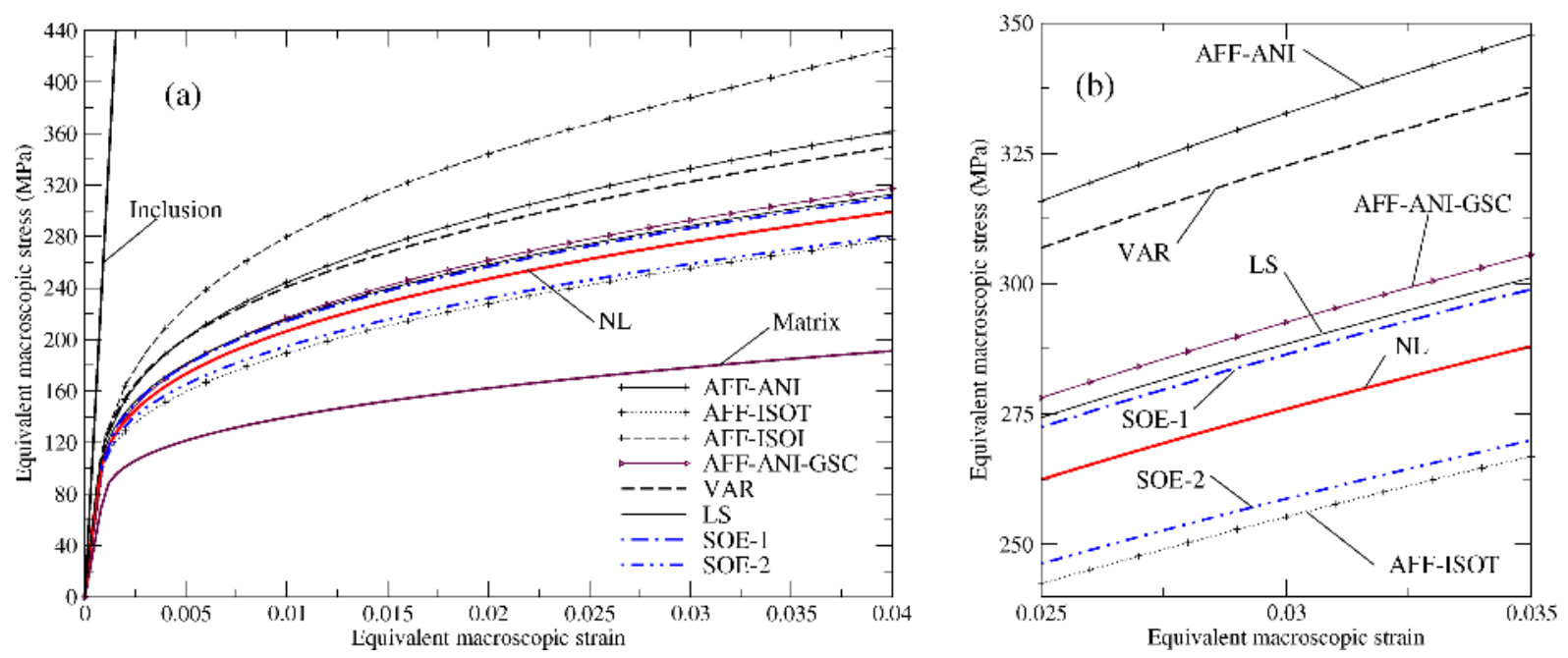

Fig. 1. Macroscopic tensile curves of a two-phase composite for different linearization procedures. Inclusion volume fraction $f_{p}=30 \%$, inclusion elastic properties: $E_{p}=400 \mathrm{GPa}, v_{p}=0.2$. Matrix elastic properties: $E_{m}=75 \mathrm{GPa}, v_{m}=0.3$; matrix power-law properties: $m=0.3895$, $\varepsilon_{0}=100 \%, \sigma_{0}=416 \mathrm{MPa}$, with a threshold stress $\sigma_{y}=75 \mathrm{MPa}$. Full curves (a) and zoom (b).

Fig. 1. Courbes macroscopiques de traction des différentes procédures de linéarisation d'un composite biphasé. Fraction volumique d'inclusion $f_{p}=30 \%$, propriétés de l'inclusion élastique : $E_{p}=400 \mathrm{GPa}, v_{p}=0,2$. Propriétés de la matrice : modules d'élasticité : $E_{m}=75 \mathrm{GPa}, v_{m}=0,3$; loi puissance de la matrice : $m=0,3895, \varepsilon_{0}=100 \%, \sigma_{0}=416 \mathrm{MPa}$, avec une contrainte seuil $\sigma_{y}=75 \mathrm{MPa}$ (a) zoom (b).

is noteworthy that a too hasty comparison based on two different microstructures could have led to a more optimistic evaluation: when the affine linearization procedure is associated with the generalized self consistent linear scheme [17] - known to provide a good estimate for a microstructure described by Hashin's composite spheres assemblage - the corresponding estimate referred to as AFF-ANI-GSC leads to a macroscopic response which is softer and better than the AFF-ANI estimate. The variational approach [2,13] predicts a slightly softer response. Again, the accurate comparison between the VAR approach and the exact solution shows a larger discrepancy than the one reported in [13] where the microstructures are different. This illustrates clearly the bias introduced in the comparisons, either by the change of microstructure, or by the approximation induced by the linear homogenization scheme. The simplified AFF-ISOT formulation, based on an isotropic approximation of the tangent modulus, softens the macroscopic response. However, this softening is so strong that the macroscopic response is significantly below the reference nonlinear solution. This result qualifies the conclusions of [7] where again the comparisons rely on different microstructures: a periodic nonlinear solution and a Mori Tanaka linear modeling approach for the LCC. The main advantage to approximate the phases behavior of the LCC by isotropic constitutive laws lies in the fact that the homogenization procedure is much more simple to put into practice. The other isotropic version of the affine approach AFF-ISOI provides a far too stiff response. Both former results suggest however the possibility to define an intermediate linearization procedure between the AFF-ISOT and the AFF-ISOI formulations which would be more efficient and still easy to implement. The two variants of the second order formulation turn out to be closer to the exact solution, SOE-1 being still too stiff but SOE-2 too soft. This confirms the great improvement due to the integration of local field fluctuations in these theories, with the price of a higher complexity. However, these results suggest also that the modifications introduced in the newer formulation might still not be optimal, since the softening they induce is too strong: in terms of accuracy, the SOE-2 formulation does not seem significantly to improve on the initial SOE-1, at least in this particular case. The LS formulation provides a macroscopic response which is nearly the same as the one derived by the initial second-order approach. This probably results from the similarity of the additional term for the polarization introduced in the LS approach and the expression of the difference between the affine and the SOE-1 estimates for the overall stress (see [3]). It is noteworthy that the LS and SOE-1 formulations are very close to the exact solution and give the best result for this particular case, an additional advantage of the LS formulation being its unambiguous definition of the local stress field. At last, let us note that other calculations carried out with different values of $m$ ranging from 0.05 to 1 showed the same general trends. 
In conclusion, note that the obtained results focused the evaluation on the macroscopic stress-strain curves. In view of comparing the respective performances of the different linearization procedures more thoroughly, it is necessary to enrich the present methodology and to take into account other informations such as the local mechanical fields. For instance, the local distributions of the strain and the stress fields deriving from the different aforementioned linearization schemes may be compared to the local reference fields [18]. Moreover, we expect these comparisons on the local scale to be helpful to define a criterion for the choice of an 'optimized' linearization procedure. At last, the present methodology can also be applied to other loading conditions, to some other microstructures such as porous media and to some other classes of constitutive behavior such as elasto-plasticity or elasto-visco-plasticity for which no bound giving an efficient reference information on the exact solution is still available. These extensions are currently under progress.

\section{References}

[1] P. Ponte Castañeda, P. Suquet, Nonlinear composites, Adv. Appl. Mech. 34 (1998) 171-302.

[2] P. Ponte Castañeda, The effective mechanical properties of nonlinear isotropic composites, J. Mech. Phys. Solids 39 (1991) $45-71$.

[3] R. Masson, M. Bornert, P. Suquet, A. Zaoui, An affine formulation for the prediction of the effective properties of nonlinear composites and polycrystals, J. Mech. Phys. Solids 48 (2000) 1203-1227.

[4] P. Ponte Castañeda, Exact second-order estimates for the effective mechanical properties of nonlinear composite materials, J. Mech. Phys. Solids 44 (1996) 827-862.

[5] M. Bornert, R. Masson, P. Ponte Castañeda, A. Zaoui, Second-order estimates for the effective behaviour of viscoplastic polycrystalline materials, J. Mech. Phys. Solids 49 (2001) 2737-2764.

[6] P. Ponte Castañeda, Second-order homogenisation estimates for nonlinear composites incorporating field fluctuations: I - Theory, J. Mech. Phys. Solids 50 (2002) 737-757.

[7] J.L. Chaboche, P. Kanouté, Sur les approximations «isotrope » et «anisotrope » de l'opérateur tangent pour les méthodes tangentes incrémentale et affine, C. R. Mécanique 331 (2003) 857-864.

[8] N. Lahellec, P. Suquet, Nonlinear composites: a linearization procedure, exact to second-order in contrast and for which the strain-energy and affine formulations coincide, C. R. Mécanique 332 (2004) 693-700.

[9] M. Bornert, P. Ponte Castañeda, Second-order estimates of the self-consistent type for viscoplastic polycrystals, Proc. Roy. Soc. London Ser. A 454 (1998) 3035-3045.

[10] P. Ponte Castañeda, P. Suquet, On the effective mechanical behavior of weakly inhomogeneous nonlinear composites, Eur. J. Mech. A Solids 14 (1995) 205-236.

[11] H. Moulinec, P. Suquet, Intraphase strain heterogeneity in nonlinear composites: a computational approach, Eur. J. Mech. A Solids 22 (2003) 751-770.

[12] T. Kanit, S. Forest, I. Galliet, V. Mounoury, D. Jeulin, Determination of the size of the representative volume element for random composites: statistical and numerical approach, Int. J. Solids Struct. 40 (2003) 3647-3679.

[13] P. Suquet, Overall properties of nonlinear composites: a modified secant moduli theory and its link with Ponte Castañeda's nonlinear variational procedure, C. R. Mécanique 320 (1995) 563-571.

[14] J.C. Michel, H. Moulinec, P. Suquet, Composites périodiques, in : M. Bornert, T. Bretheau, P. Gilormini (Eds.), Homogénéisation en mécanique des matériaux, tome 1, Hermes Science Publications, 2001, pp. 57-94.

[15] L.J. Walpole, Elastic behavior of composite materials: theoretical foundations, in: Adv. Appl. Mech., vol. 21, Academic Press, New York, 1981, pp. 160-242.

[16] P. Suquet, M. Bornert, Calcul tensoriel et élasticité, in: M. Bornert, T. Bretheau, P. Gilormini (Eds.), Homogénéisation en mécanique des matériaux, tome 2, Hermes Science Publications, 2001, pp. 171-202.

[17] R.M. Christensen, K.H. Lo, Solution for effective shear properties in three phase sphere and cylinder models, J. Mech. Phys. Solids 27 (1979) 315-330.

[18] A. Rekik, M. Bornert, F. Auslender, A. Zaoui, Evaluation précise des procédures de linéarisation en homogénéisation nonlinéaire, in: 17ème Congres Français de Mécanique, Troyes, France, 2005. 